\title{
Clasificación y Modelos Animales de Status Epilepticus
}

Fridha Viridiana Villalpando Vargası ${ }^{*}$, Laura Guadalupe Medina Ceja, Sofía Rodríguez Mercado y Consuelo Ventura Mejía

\author{
1Laboratorio de Neurofisiología, Departamento de Biología Celular y Molecular, División de Cien- \\ cias Biológicas y Ambientales, Centro Universitario de Ciencias Biológicas y Agropecuarias, \\ Universidad de Guadalajara. Camino Ramón Padilla Sánchez \\ No. 2100 Nextipac, Zapopan, Jalisco C.P.45200. \\ ${ }^{*}$ Autor para correspondencia: \\ dra.villalpando@hotmail.com, viridiana.villalpando@academicos.udg.mx
}

\section{Resumen}

El estatus epilepticus (SE) representa la forma más severa de crisis epiléptica, da lugar a crisis anormalmente prolongadas $y$, dependiendo del tipo y duración de las crisis, conlleva consecuencias a largo plazo incluyendo alteraciones de las conexiones neuronales, muerte y daño neuronal. El tratamiento del SE representa un reto debido a la escasez de fármacos efectivos. El conocimiento y domi-nio del SE por el personal médico es de suma importancia para establecer un abordaje terapéutico certero y eficaz, a este respecto la clasificación del SE de acuerdo a sus cuatro parámetros: semiología, etiología, correlato electroencefalgráfico y edad, son fundamentales para establecer decisiones terapéuticas. Por su parte, la investigación básica permite el desarrollo de nuevas estrategias terapéuti-cas que permitan investigar de manera segura tanto los mecanismos que inducen el establecimiento del SE como su abordaje clínico-quirúrgico. En el presente trabajo se describirá la actual clasificación del SE de acuerdo a la Liga Internacional contra la Epilepsia (ILAE, por sus siglas en inglés: International Ligue Against Epilepsy) y los modelos animales de inducción química del SE más ampliamente utilizados: pentilentetrazol, pilocarpina y ácido kaínico.

Palabras clave: epilepsia, anticonvulsivos, pentilentetrazol, pilocarpina, ácido kaínico.

\section{Abstract}

The Status epilepticus (SE) represents the most severe form of epileptic seizures, which lead to abnormally prolonged seizures, depending on the type and duration of seizures. It can have long-term consequences, including alteration of neuronal networks, neuronal death and injury. The treatment of SE represents a challenge due to the scarcity of effective drugs. Knowledge and dominance of the SE by the medical personnel is of utmost importance to establish an accurate and effective therapeutic approach, in this res-pect the SE classification according to its four pa-rameters: semiology, etiology, electroencepha-lographic correlate and age, are fundamental to establishing therapeutic decisions. On the other hand, basic research allows the development of new therapeutic strategies that allow to safely investigate both the mechanisms that induce SE establishment and its clinical-surgical approach. In this paper we will describe the current SE classification according to the International League Against Epilepsy (ILAE) and most widely used ani-mal models of chemical induction of SE: pentyle-netetrazole, pilocarpine and kainic acid.

Key words: epilepsy, anticonvulsants, pentylenetetrazole, pilocarpine, kainic acid. 


\section{Introducción}

Los pacientes epilépticos se ven afectados por los diversos efectos colaterales a los fármacos anticonvulsivos en un $40 \%$ y hasta un $30 \%$ de presentan epilepsia refractaria al tratamiento que puede conducir a la presentación del status epilepticus (SE), que es la presentación más severa de crisis epiléptica (Newton \& García 2012). Por lo que ha surgido la necesidad de desarrollar nuevos abordajes terapéuticos para tratar la epilepsia con el objetivo de ofrecer una mayor efectividad anticonvulsiva y disminuir los efectos colaterales; a este respecto la clasificación de del SE así como modelos que permitan evaluar la efectividad y seguridad de los fármacos ha sido una de las bases que permite el desarrollo de nuevas estrategias terapéuticas.

EI SE fue definido por McCandless (2012) como crisis recurrentes sin recuperación que persis-ten por 30 minutos o más. Sin embargo, Trinka et al. (2015) establecieron que el SE es una condición que resulta de la falla de los mecanismos responsables de la terminación de la crisis o del inicio de mecanismos que conducen a convulsiones anormalmente prolongadas (después del punto de tiem-po t1). Es una afección que puede tener consecuencias a largo plazo (después del punto de tiempo t2), incluida la muerte neuronal, la lesión neuronal y la alteración de las redes neuronales, según el tipo y la duración de las convulsiones.

Cuadro 1. Dimensiones operacionales temporales. Tiempo uno (tl) que indica el momento en que debe iniciarse el tratamiento de emergencia del status epilepticus (SE) y del tiempo dos (t2) que indica el momento en el que se pueden esperar consecuencias a largo plazo.

\begin{tabular}{|c|c|c|}
\hline Tipo de SE & $\begin{array}{l}\text { t1: cuando es probable que } \\
\text { una convulsión se prolongue, } \\
\text { lo que lleva al } \\
\text { establecimiento de una } \\
\text { actividad convulsiva } \\
\text { continua }\end{array}$ & $\begin{array}{l}\text { t2: cuando una convulsión } \\
\text { puede causar } \\
\text { consecuencias a largo } \\
\text { plazo (incluyendo lesión } \\
\text { neuronal, muerte } \\
\text { neuronal, alteración de } \\
\text { redes neuronales y déficits } \\
\text { funcionales) }\end{array}$ \\
\hline SE tónico-clónico & $5 \mathrm{~min}$ & $30 \mathrm{~min}$ \\
\hline $\begin{array}{l}\text { SE focal con alteraciones } \\
\text { de la conciencia }\end{array}$ & $10 \mathrm{~min}$ & $>60 \mathrm{~min}$ \\
\hline SE de ausencia & $10-15 \min *$ & Desconocido \\
\hline
\end{tabular}

*La evidencia de la ventana temporal es limitada y puede que sea necesario modificar este dato en el futuro (modificado de Trinka et al. 2015). 
EI SE es la segunda emergencia neurológica, seguida de la enfermedad cerebrovascular aguda. A nivel mundial ocurren 1 millón de eventos, con una incidencia anual que va de los 18 a los 20 casos por cada 100,000 habitantes (Chin et al 2006) y se asocia a una tasa de mortalidad de 3 al 24.4\% (Her-nández-Domínguez et al, 2017; Delaj et al, 2016; Barzegar et al, 2015; Owolabi et al, 2014; Alvarez et al, 2012; Chin et al, 2006). En los Estados Unidos de Norteamérica, se estima que el 0.5\% de los pacientes epilépticos presentan un SE, siendo más propensos los niños mayores de 1 año de edad. Se estima que el $70 \%$ de los pacientes presentarán un SE en alguna etapa de su vida y de estos el 41\% presentará crisis futuras (Hauser 1990; Hesdorffer et al. 1998; Trinka et al. 2012).

\section{Clasificación del status epilepticus}

EI SE se clasifica considerando cuatro ejes principales:

\section{Cuadro 2. Clasificación del status epilepticus (SE) según la semiología}

A. Síntomas motores prominentes

A.1. SE convulsivo (SEc; también conocido como tónico-clónico)

A.1.a. Convulsivo generalizado

A.1.b. Inicio focal que evoluciona a un SEc generalizado.

A.1.c. Se desconoce si es focal o generalizado

A.2. SE mioclónico (tirones mioclónicos epilépticos prominentes)

A.2.a. Con coma

A.2.b. Sin coma

\section{A.3. Motor focal}

A.3.a. Convulsiones motoras focales repetidas (Jacksoniano)

A.3.b. Epilepsia parcial continua

A.3.c. SE adversivo

A.3.d. SE oculoclónico

A.3.e. Paresis ictal

A.4. SE tónico

A.5. SE hipercinético
B. Sin síntomas motores prominentes

B.1. SE no convulsivo con coma (también llamado SE sutil)

B.2. SE no convulsivo sin coma

B.2.a. Generalizado

B.2.a.a. SE típico de ausencia

B.2.a.b. SE no típico de ausencia

B.2.a.c. SE miclónico de ausencia

B.2.b. Focal

B.2.b.a. Sin alteraciones de la conciencia (aura continua, con síntomas autonómicos, sensoriales, olfatorios, visuales, gustativos, auditivos, emocional/ psíquico/experiencial)

B.2.b.b. SE afásico

B.2.b.c. Con alteración de la conciencia

B.2.c. Se desconoce si es focal o generalizado

B.2.c.a. SE autonómico 
Cuadro 3. Clasificación del status epilepticus (SE) según la etiología. Las causas pueden ser estructurales, metabólicas, inflamatorias, infecciosas, tóxicas o genéticas y pueden subdividirse de acuerdo a su asociación temporal en agudas, remotas o progresivas

Origen conocido

Origen conocido

Agudo (ejemplo: accidente cerebrovascular, Remoto (post-traumático, post-encefalítico, intoxicación, malaria, encefalitis, etc.) post-accidence cerebrovascular, etc)

Progresivo (ejemplo: tumor cerebral, SE asociado a síndromes electroclínicos demencias, Enfermedad de Lafora, etc) definidos (epilepsia infantil benigna, síndrome de Lennox-Gastau)

Origen desconocido (ejemplo: criptogénica)

El SE en sus variantes formas tiene una gran cantidad de causas, que son enlistadas y actualizadas continuamente por clínicos, estas bases se pueden encontrar en la página de la International Ligue Against Epilepsy (ILAE.org) (modificado de Trinka et al. 2015). 


\section{Cuadro 4. Clasificación del status epilepticus (SE) según el correlato electroencefalográfico}

1. Localización: generalizada (incluye 4. Características relacionadas con el patrones sincrónicos bilaterales), tiempo: prevalencia, frecuencia, duración, lateralizada, bilateral independiente o índice y patrón de duración diaria, inicio multifocal (súbito vs gradual) y evolutivo, fluctuante o estático

2. Nombre del patrón: descargas periódicas, 5.Modulación: inducido por un estímulo vs actividad delta rítmica, espiga-onda, espontáneo poliespiga

3. Morfología: agudeza, número de fases Efecto de la intervención (medicación) en el (morfología trifásica), amplitud absoluta y EEG relativa, polaridad

Correlato electroencefalográfico: a pesar de que el EEG está sobre cargado con el movimiento y artefactos musculares en el SE convulsivo, es indispensable para el diagnóstico del SE no convulsivo. No existen criterios EEG en SE, los autores sugieren la siguiente terminología para describir los patrones EEG del SE (modificado de Trinka et al. 2015). 
Cuadro 5. Clasificación del status epilepticus (SE) de acuerdo a la edad. Existen SE que se presentan en pacientes con síndromes electroclínicos asociados a la edad que ocurren por causas directamente ligada a diferentes grupos de edad, a continuación, se enlistan algunos ejemplos.

1. Neonatal (0-30días) en neonatos puede ser difícil de identificar o sutil

2. Infantil (1mes a 24 meses) ejemplo: SE clónico (Síndrome de Otahara, Síndrome de West), SE miclónico en el síndrome de Dravet, SE focal, SE febril.

3. En la niñez (2 a 12 años) ejemplos: SE autonómico en la epilepsia occipital infantil benigna de inicio temprano (Síndrome de Panayiotopoulos), SE no convulsivo en síndromes y etiologías específicas de la niñez, SE tónico en el Síndrome de LennoxGastaut, SE mioclónico en epilepsia mioclónicas progresivas, SE eléctrico durante el sueño de ondas lentas, SE afásico en el Síndrome de Landau-Keffner.

4. Adolescencia y adultez ( $>12-59$ años) ejemplo: SE mioclónico en epilepsia mioclónica juvenil, SE de ausencia en epilepsia de ausencia juvenil, SE mioclónico en Síndrome de Down.

5. En el adulto mayor ( $>60$ años) ejemplo: SE mioclónico en Enfermedad de Alzheimer, SE no convulsivo asociado a la Enfermedad de Creutzfeldt-Jakob, estado de ausencia de novo (o recurrente) en la etapa de adulto mayor.

Cabe destacar que estos ejemplos son más frecuentes en estos grupos de edad, pero no son exclusivos (modificado de Trinka et al. 2015). 
1. Semiología

2. Etiología

3. Correlato electroencefalográfico (EEG)

4. Edad

Idealmente, cada paciente debe categorizarse de acuerdo con cada uno de los cuatro ejes. Sin embargo, se reconoce que esto no siempre será posible. En la presentación inicial, la edad aproximada del paciente y la semiología serán inmediatamente evaluables. La etiología será aparente con menos frecuencia y puede llevar tiempo identificarla. También se reconoce que las grabaciones de EEG no estarán disponibles en muchas configuraciones, particularmen-te en la presentación. A pesar de estas limitantes, el EEG afectará la elección y la agresividad del tratamiento, el pronóstico y los enfoques clíni-cos, por lo que se debe buscar un EEG siempre que sea posible y tan pronto como sea posible. De hecho, algunas formas de SE solo pueden ser diagnosticadas de manera confiable por EEG (Bauer \& Trinka 2010, Trinka et al 2015).

Cuero et al (2015) y Datar (2017), sugieren una subclasificación del SE en la que aquellas convulsiones continuas o recurrentes con una duración mayor a 24 horas tras el inicio del tratamiento anticonvulsivo, incluyendo los casos en que las convulsiones recurren una vez que pasa el efecto anestésico de los fármacos, se deben deno-minar Status epiléptico super refractario (SESR). Diversos estudios establecen que incidencia de SESR en pacientes con EE va del 20.3 al $40.15 \%$ y es considerado una variable que aumenta signi-ficativamente el riesgo de secuelas neurológicas y muerte (Atmaca et al, 2017; Hernández-Domín-guez et al, 2017; Delaj et al, 2016; Barzegar et al, 2015; Owolabi et al, 2014)

\section{Fisiopatología del status epilepticus}

Las crisis son originadas por una disrupción temporal de la función cerebral debido a descargas eléctricas excesivas y anormales de neuronas. Dicha actividad es el producto neto de procesos bioquímicos a niveles celulares y sub-celulares que ocurren a lo largo de las re- des neurales. Las crisis generalmente involu-cran interacciones entre estructuras corticales y subcorticales (Blumenfeld 2003; Dichter 2009; Fisher 2005). A nivel celular, las dos característi-cas distintivas de la actividad epiléptica son la hiperexcitabilidad e hipersincronía neuronal. La hiperexcitabilidad es una respuesta anormal (por ejemplo, por un umbral inferior) de la neurona al estado excitador; una neurona hiperexcitable tiende a disparar ráfagas de múltiples potenciales de acción en lugar de sólo uno o dos. Por otra parte, la hipersincronización neuronal se refiere al reclutamiento de un gran número de neuro-nas vecinas en un modo de disparo anormal; por lo que las crisis son un fenómeno de red que requiere la participación de neuronas disparando de forma sincrónica. Las técnicas electroencefalográficas (EEG) convencionales pueden detectar áreas corticales que demuestran descargas hipersincrónicas en forma de ondas agudas interictales o picos, observables en diferentes modelos experimentales (Bragin et al. 2002; Buzsaki et al. 1992; Grenier et al. 2001; Ylinen et al. 1995).

Desde el punto de vista neuroquímico, la crisis son el resultado de un desequilibro entre la actividad excitadora principalmente media-da por el neurotransmisor glutamato (Glu) y la actividad inhibidora principalmente mediada por el neurotrasmisor ácido $\gamma-$ aminobutírico (GABA) (Medina-Ceja et al. 2000; Rowley et al. 1995; Tapia et al. 1999).

Las descargas eléctricas anormales focales pueden difundirse a través de mecanismos sinápticos y no sinápticos a nivel local (crisis parciales o focales), o bien, propagarse distalmen-te tanto a las estructuras subcorticales como a toda la corteza por medio de las vías comisura-les (crisis focal con generalización secundaria). En contraste con las crisis generalizadas (generalización primaria), que inician con descargas eléctricas anormales en ambos hemisferios cerebrales de manera simultánea (Stafstrom 2010). 

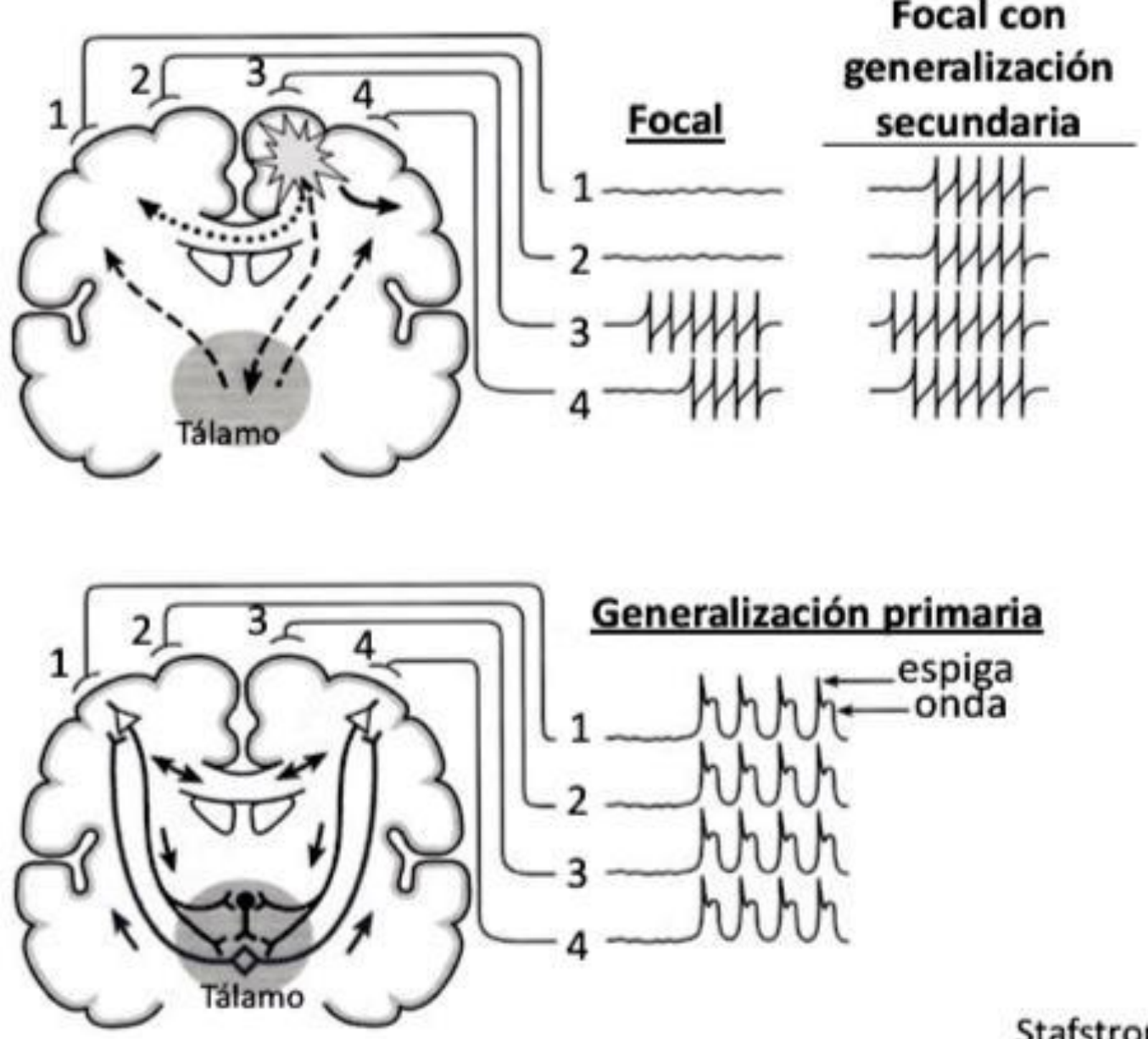

Stafstrom y cols. 2004

Figura 1. Cortes coronales del cerebro que representan los tipos de convulsiones y posibles vías de propagación. A) Área focal de hiperexcitabi-lidad (debajo del electrodo 3) y la propagación a la neocorteza adyacente (flecha continua debajo del electrodo 4), vía cuerpo calloso (línea puntea-da) u otras vías comisurales del hemisferio cerebral contralateral o vía subcortical (i.e., tálamo; flechas discontinuas hacia arriba). Los patrones EEG muestran actividad eléctrica del cerebro bajo los electrodos 1 al 4 . La actividad epileptiforme focal es máxima en el electrodo 3 y también se ob-serva en el electrodo 4 (trazos en la izquierda). Si la crisis se generaliza secundariamente, la actividad puede sincronizarse en todos los electrodos, después de un retraso temporal (trazos en la derecha). B) Las crisis primarias generalizadas inician de manera simultánea en ambos hemisferios. Los patrones EEG característicos son espigas-ondas bilaterales sincronizadas, es generado por interacciones recíprocas entre la corteza y el tála-mo, con una rápida propagación vía cuerpo calloso (CC), contribuyendo a la rápida sincronización bilateral. Un tipo de neurona talámica (neurona en negro) es una célula inhibitoria GABAérgica que muestra la actividad intrínseca de marcapasos. Las neuronas corticales (triángulos) envían impulsos tanto a los relevos talámicos (rombo) como a las neuronas inhibitorias, estableciendo oscilaciones excitadoras y actividad de inhibición,

dando lugar a los patrones espiga-onda rítmica en el EEG (modificado de Stafstrom, 2004).

Las crisis de generalización primaria involucran las conexiones tálamo-corticales recíprocas. Las ma-nifestaciones de dicha actividad epiléptica generalizada pueden variar de breve alteración de la conciencia (como en una crisis de ausencia) hasta movimientos espasmódicos rítmicos de todas las extremidades, acompañadas por la pérdida de la postura y la conciencia, como en una convulsión tónico-clónica generalizada (Stafstrom 2010). 


\section{Modelos de status epilepticus}

Es importante reconocer que los modelos animales de SE, se han utilizado para dilucidar mecanismos neuronales de generación y mantenimiento de esta patología (Engel 2001). Además, se han empleado en la investigación específicamente diseñada para planear nuevos enfoques y pruebas de fármacos anticonvulsivos; incluso, los modelos animales resultan indispensables para comprobar la eficacia y seguridad de estos (Engel \& Schwartzkroin 2006)

Existen diversos tipos de inducción del status epilepticus: químico (por ejemplo: PTZ, pilocarpina, penicilina, 4-aminopiridina), eléctri-co (ejemplo: estimulación eléctrica) y físico (por ejemplo: hipertermia y postraumatismo craneoncefálico). En el presente trabajo nos centraremos en los modelos de inducción de crisis química.

Modelo de pentilentetrazol

El pentilentetrazol (PTZ) es un derivado del tetrazol (Stone 1970), que originalmente se utilizaba como un cardio-estimulante. Se ha demostrado que presenta acción convulsiva en ratón, rata, gatos y primates (Vernadakis \& Woodbury 1969a, b; Reinhard \& Reinhard 1977; Swinyard et al. 1989). También es conocido como Cardia-zol, Metrazol, Pentetrazol, Pentazol, entre otros. Actúa como antagonista no competitivo de los receptores GABAA, interacciona con el sitio de unión a t-butil-biciclo-fosforotionato

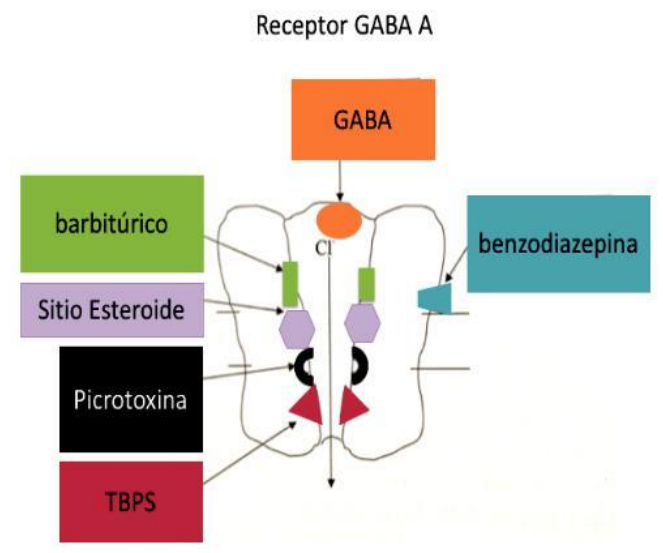

Figura 2. Receptor GABAA. El pentilentetrazol interactúa con el sitio de unión de t-butil-biciblo-fosforotionato (TBPS).
(Hansen et al. 2004; Olsen 1981; Ramanjaneyu-lu \& Ticku, 1984). Además, es uno de los quími-cos convulsivantes de primera elección para la evaluación de drogas anticonvulsivas (Hansen et al. 2004; Kupferberg 2001). Las manifestaciones conductuales y EEG causadas por PTZ lo hacen un modelo de crisis generalizadas, cuando se emplean dosis bajas $(20-30 \mathrm{mg} / \mathrm{Kg}$ de peso). Pue-de utilizarse como un modelo de crisis de ausen-cia; en dosis altas $(40-100 \mathrm{mg} / \mathrm{Kg}$ de peso) genera crisis tónico-clónicas (Cortez \& Snead 2006; Zhao

$\&$ Holmes 2006; Erdogan et al. 2014).

El efecto de PTZ en sistema nervioso central es inespecífico; sin embargo, se sugiere que las crisis inducidas por este fármaco están mediadas por estructuras cerebrales como neocorteza (Ben-Ari 1981a,b; Browing 1985), extensión clónica se modula en regiones del diencéfalo y el tallo cerebral, incluyendo la formación reti-cular, el bulbo y la sustancia gris central (Yone-kawa 1980; Browing 1985; Miller 1987). El PTZ se disuelve en solución salina fisiológica o agua, por lo que puede ser administrada subcutánea (s.c.), intraperitoneal (i.p., la más utilizada) 0 intracerebroventricular (i.c.v., la menos utilizada). La dosis que produce crisis en el $50 \%\left(C_{50}\right)$ de las ratas, en el caso de ratas macho de la cepa Wistar, depende de la edad; por lo tanto, las crisis tónico-clónicas son observadas durante todo el desarrollo mientras que las crisis clónicas son limitadas durante las dos primeras semanas postnatal (Velísek et al. 1992). Los niveles necesarios de $\mathrm{CD}_{50}$ por vía i.c.v. e i.p. son menores en comparación con los de la vía s.c. (Fisher 1989). Para fines prácticos, las dosis alrededor de $100 \mathrm{mg} / \mathrm{Kg}$ i.p. o s.c. son más frecuentemente utilizadas y desarrollan convulsiones dentro de los 20 minutos posteriores a su administración.

Los cambios conductuales inducidos por PTZ son: congelamiento (arresto motor), tics mioclónicos, convulsiones clónicas y convulsiones tónico-clónicas. Los cambios conductuales son dosis dependiente, las dosis bajas pueden inducir solamente congelación asociado a la presencia de husos en el EEG, la dosis puede ir aumentando hasta más de $100 \mathrm{mg} / \mathrm{Kg}$ para generar convulsiones tónico-clónicas con patrón 
de poliespigas de gran amplitud y frecuencia

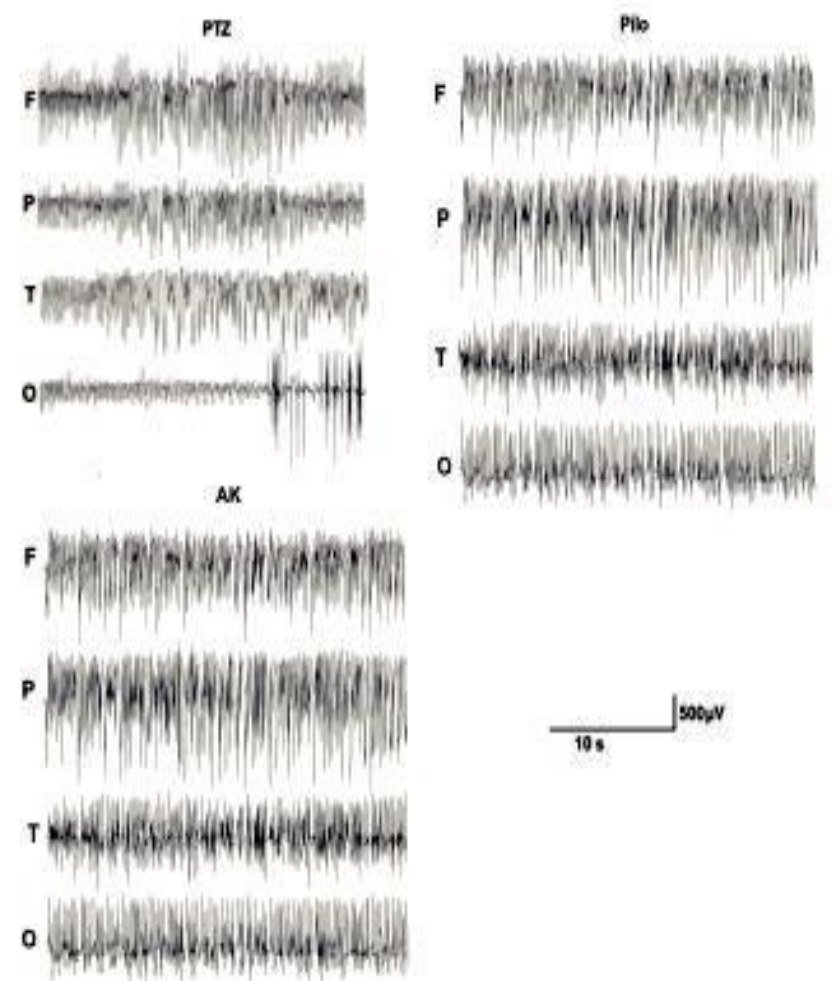

Figura 3. Composiciones de EEG representativas de trenes de descarga inducido por pentilentetrazol (PTZ), pilocarpina (pilo) o ácido kaínico (AK) de gran amplitud con patrón de poliespiga rítmica. El ex-perimento se realizó con un registro superficial en ratas Wistar adultas, con una administración de PTZ de $90 \mathrm{mg} / \mathrm{kg}$ (i.p.), pilocarpina de $360 \mathrm{mg} / \mathrm{kg}$ (i.p.) o ácido kaínico $10 \mathrm{mg} / \mathrm{kg}$ (i.p.). Abreviaturas F: frontal, $P$ : pa-rietal, $T$ : temporal, $O$ : occipital, $u V$ : microvolts, $s$ : segundos (modificada de Villalpando-Vargas \& Medina-Ceja 2015).

(Villalpando-Vargas \& Medina- Ceja 2015). Ade-más de la dosis, la edad influye en la latencia de la presentación de las convulsiones (de Casrile-vitz et al. 1971; Velísek et al. 1992; Vernadakis \& Woodbury 1969a; Weller \& Mostofsky 1995). Las drogas como la etosuximida, el clonazepam y el ácido valproico pueden suprimir las espigas EEG (Brabcová et al. 1993) y las convulsiones tónico-clónicas inducidas por PTZ (Mares et al. 1981).

\section{Modelo de pilocarpina}

El modelo de pilocarpina puede emplear-se para la inducción aguda de crisis con la culminación de un episodio de SE, o bien, como un modelo crónico de crisis espontáneas (epielptogénesis pos-SE). La inducción aguda se caracteriza por un SE de larga duración, asociado con des- cargas electroencefalográficamente sostenidas de estructuras límbicas (Turski et al. 1983a; 1984).

En roedores, la administración de pilocar-pina induce actividad epiléptica ictal e interictal; de manera inmediata después de la inyección, en registros EEG se observan ritmos theta en regiones hipocámpicas y actividad rápida de bajo voltaje en corteza; esta actividad eléctrica está relacionada con alteraciones en el comportamiento que inclu-yen acinesia, ataxia y automatismos faciales. La actividad eléctrica progresa a espigas rápidas de alto voltaje en el hipocampo que se propagan a la corteza evolucionando a crisis electrográficas, por lo tanto, la severidad de las manifestaciones físicas progresa a convulsiones motoras; el periodo ictal se repite cada 3 a 5 minutos y finalmente desen-cadena descargas sostenidas (SE) 50 a 60 minutos después de la inyección (Figura 3) (Cavalheiro et al. 2006; Fisahn et al. 1998; Van der Linden et al. 1999).

Durante el periodo agudo (posterior a la aplicación única de pilocarpina) el SE puede durar de 6 a 12 horas; una vez establecida la remisión del $\mathrm{SE}$, los animales se encuentran comatosos y ambos registros, hipocámpicos y corticales, es-tán deprimidos, con actividad de espigas de alto voltaje. Estudios metabólicos realizados durante este periodo revelan un incremento en la utiliza-ción de glucosa, principalmente en hipocampo y otras estructuras límbicas como tálamo y sustan-cia negra. Además, se observa una disminución de neuronas en dichas estructuras. El sistema colinérgico juega un papel importante en el marcaje y mantenimiento de las convulsiones, la liberación sináptica de acetilcolina (Ach) provee un impulso sostenido que mantiene a la neurona disparando (como en el SE) (Cavalheiro et al. 2006).

Además, se ha reportado que la pilocarpina induce crisis agudas en ratas Wistar (Turski et al. 1983a). Con respecto a la especificidad por edad, el modelo de pilocarpina puede inducir crisis en ratas posnatales de 11 días hasta aproximada-mente 12 meses de edad (Cavalheiro et al. 1987; 2006). Los signos conductuales que involucran crisis límbicas se intensifican y producen SE en ra-tas mayores de 11 días posnatales; sin embargo, la mayor sensibilidad para la inducción del SE por pilocarpina está entre los 15 y 21 días de edad, en 
donde los patrones de pérdida neuronal y los cambios plásticos consiguientes son más marcados en este periodo. Por lo tanto, los animales jóvenes presentan una mayor sensibilidad a la inducción aguda de crisis por pilocarpina y frecuentemente requieren dosis $30 \%$ menores a la de los animales adultos. En contraste, estos animales son menos vulnerables a los daños causados por el SE inducido por pilocarpina, así como a los cambios plásticos (Cavalheiro et al. 1987; Santos et al. 2000).

También en este modelo se ha demostrado un incremento en la tasa de utilización de norepin-efrina (NE), así como una disminución en la tasa de utilización de dopamina tanto en el periodo agudo como en el latente y crónico. Sin embargo, el incremento de la tasa de utilización de serotonina solo se ha reportado en la fase aguda (Cavalheir et al. 1994). La depleción de NE se ha asociado con un incremento de la susceptibilidad de crisis, mientras que la liberación de NE se relaciona con una pro-tección contra la propagación o inicio de las crisis (Mason \& Corcoran 1979; Maclntyre \& Edson 1982).

En contraste, en la inducción de crisis por litio más pilocarpina, antagonistas de los receptores dopaminérgicos D2 reducen el umbral de las crisis, mientras que los antagonistas D1 previenen la actividad convulsiva, demostrando que los receptores dopaminérgicos ejercen una función opuesta en la regulación de la actividad convulsiva en este modelo (Barone et al. 1991).

La fase aguda del modelo de pilocarpina se caracteriza por un incremento en la tasa de liberación de Glu en el hipocampo, esta evidencia es apoyada por un estudio en animales sometidos a un SE de larga duración (12 h) donde los sinaptosomas extraídos de los cerebros de estos animales presentaban un aumento en la liberación de Glu; lo que apoya el fenómeno excitador (Cavalhei-ro et al. 1994; Costa et al. 2004; Smolders 1997). El Glu actúa sobre los receptores ácido $\alpha$-Ami-no-3-hidroxi5-metil-4-isoxazolepropionico /kai-nato (AMPA/KA), esto permite la entrada de sodio y calcio, en consecuencia, el magnesio es removi-do de los receptores $\mathrm{N}$-metil-D-aspartato (NMDA) lo que permite una mayor entrada de calcio y la hiperexcitabilidad (Cavalheiro et al. 2006).

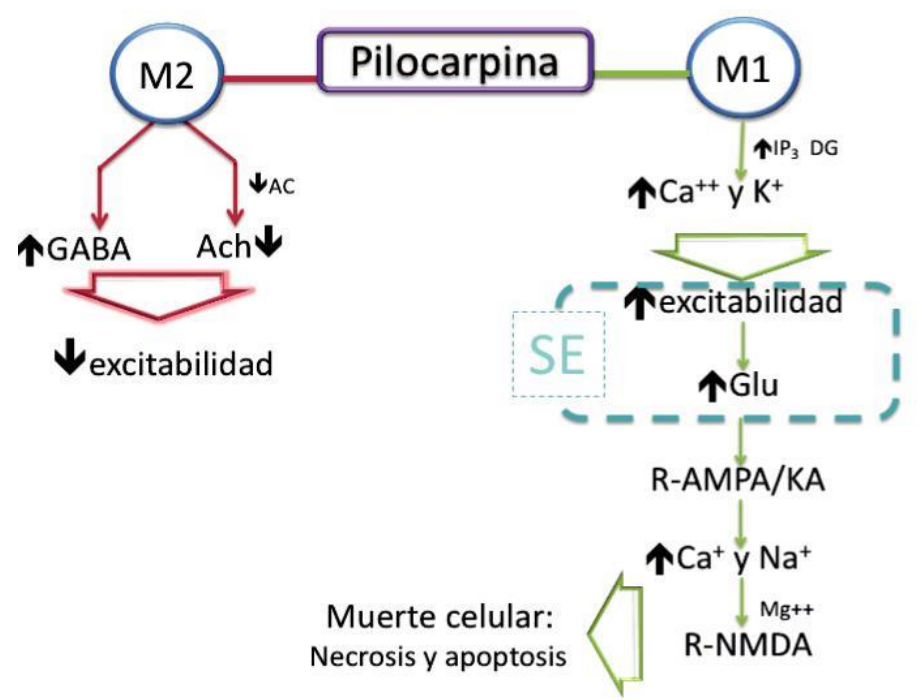

Figura 4. Mecanismo de acción de pilocarpina en los receptores muscarínicos. Pilocarpina tiene el potencial de activar los receptores muscarínicos 1 y 2. La activación de los receptores muscarínicos 1 permiten la entrada calcio y potasio a la célula, lo cual favorece la excitabilidad y la inducción del SE con la consecuente liberación de Glu, esto permite mantener la excitabilidad. Además, Glu activa los receptores AMPA/KA que permiten la entrada de sodio y calcio, en consecuencia, el magne-sio es removido de los receptores NMDA y permite un influjo mayor de calcio y sodio, esto favorece la excitabilidad y muerte celular. Abrevia-ciones: $A C$ adenil ciclasa, Ach acetilcolina, Ca+ calcio, DG diacilglicerol, GABA ácido $\gamma$ aminobutírico, Glu glutamato, IP3 inositol 1,4,5-trifosfa-to, K+ potasio, M1 receptor muscarínico tipo 1, M2 receptor muscarínico tipo 2, Mg++ magnesio, Na+ sodio, R-AMPA/KA receptores ácido al-fa-amino-3-hidroxi-5metil-4-isoxazolpropiónico (AMPA) y receptores kainato (KA), R-NMDA receptor $N$-metil-D-aspartato, $S E$ status epilep-ticus (modificado de Cavalheiro et al. 2006).

Este aumento de concentración de calcio intracelular induce la activación de lipasas, proteasas y nucleasas, generando muerte celular por necrosis, apoptosis o una combinación de ambas (Cavalheiro et al. 2006). Funke et al. (2003) demuestran la participación de otros receptores de Glu en el fenómeno excitador durante la fase aguda, al encontrar un incremento en la expresión del receptor de Glu metabotrópico 1 (mGluR1) a lo largo de la formación hipocampal.

Además, se ha demostrado que el hipo-campo también mantiene la excitabilidad debido a una disminución en la actividad de la bomba de sodiopotasio ATPasa durante el periodo agudo (Fernandes et al. 1996). Incluso, las enzimas encar-gadas de la extrusión de calcio de las neuronas a través de la membrana plasmática (PMCAs) y las ATPasas del sub-tipo SERCA2b distribuida en el ce-rebro que se encarga de la retención de calcio en el sarcoplasma o retículo endoplásmico, se incre-mentan después de 1 hora del SE, lo que demuestra 
un intento para controlar la excitabilidad tisular y evitar los procesos de muerte celular inducidos por calcio previamente descritos (Funke et al. 2003).

Diversos estudios establecen que la pilocarpina activa los receptores muscarínicos M1 como M2, los cuales son críticos para la inducción del SE, tanto para la administración sistémica (Hamilston et al. 1997) como para la administración intracerebroventricular (i.c.v.) o intrahipocampal (i.h.) (Croiset \& De Wied 1992; Millian et al. 1993). Al actuar sobre los M2, la adenilato ciclasa (AC) es inhibida, disminuyendo la liberación de Ach y la excitabili-dad neuronal (Smolders 1997).

Por otra parte, la unión de pilocarpina a M1, activa la fosfolipasa $\mathrm{C}$, produciendo diacilglicerol (DG) e inositol trifosfato (IP3), lo que altera la corriente de calcio y potasio e incrementa la excitabilidad neuronal (Figura 4) (Segal 1988).

\section{Modelo de ácido kaínico}

El ácido kaínico (AK) es un análogo del áci-do glutámico, que induce crisis límbicas y daño neuronal subsecuente, principalmente en las re-giones CA1 y CA3 del hipocampo, amígdala y cor-teza perirrinal y entorhinal; asimismo produce gliosis (Miller et al.1990; Sampieri et al. 2011).

El modelo de AK como el de pilocarpina presenta al menos tres etapas: (1) las horas iniciales a lo largo del SE, fase aguda; (2) los días o semanas libres de crisis, fase latente; (3) el inicio, desarrollo gradual e incremento progresivo de las crisis, fase crónica (Curia et al. 2008), cuando el objetivo es el estudio del SE, el enfoque principal es estudiar la fase aguda.

El AK puede disolverse en solución salina fisiológica; sin embargo, el pH resultante es ácido, por lo que se utiliza una solución amortiguadora de fosfatos ( $\mathrm{pH}$ 7.4). Las vías de administración son intraperitoneal (i.p.), subcutánea (s.c.), i.c.v. o i.h. Las dosis dependen del estado de maduración del animal, usualmente las dosis para generar convulsiones y SE en ratas adultas son de 10 a $14 \mathrm{mg} / \mathrm{Kg}$, en ratas jóvenes es de de 1 a $4 \mathrm{mg} /$ $\mathrm{Kg}$ (Velísek 2006). Los tratamientos pueden administrarse en una sola dosis alta (i.e. $8-15 \mathrm{mg} / \mathrm{Kg}$ ) o en dosis bajas múltiples (i.e. $2.5-5 \mathrm{mg} / \mathrm{Kg}$ ) (Dudek et al. 2006). Uno de los principales problemas con las dosis únicas es la alta mortalidad, en algunos casos se puede detener el SE con benzodiacepinas después de un tiempo determinado (i.e. $30 \mathrm{mi}$ nutos), pero algunos animales pueden morir antes de la inyección de benzodiacepinas (Tremblay et al. 1984). Otro problema frecuente con las dosis únicas es que algunos animales no presentan SE (Tremblay et al. 1984; Pirttila et al. 2001).

Por otro lado, las inyecciones intracranea-les de AK requieren de anestesia y técnicas estereotáxicas que por su naturaleza tienen la ventaja de generar un daño más localizado; incluso Nadler (1981) reportó que cuando el $\mathrm{AK}$ es administrado en una región específica de la región límbica, las crisis inician en esa área. En cuanto a la dosis de administración se han reportado $0.4 \mu \mathrm{g} / 0.2 \mu \mathrm{l}$ de $\mathrm{AK}$ en solución salina

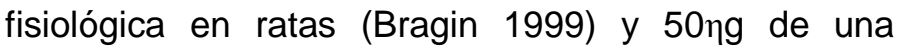
solución de $20 \mathrm{mM}$ de $\mathrm{AK}$ en $0.9 \% \mathrm{NaCl}$ en ratones (Riban 2002) ambas por una vía i.h.

La administración sistémica de AK produce hipo-actividad durante 20-30 minutos (Ben-Ari et al. 1981), posteriormente se presentan automatis-mos específicos de la edad; en ratas de 2 semanas de edad prevalece el rascado (Albala et al. 1984; Cherubini et al. 1983; Tremblay et al. 1984; Velísková et al. 1988), en la tercera semana empiezan a emerger "sacudidas de perro mojado", su frecuencia incrementa con la edad (Velísek 2006). Las convulsiones clónicas pueden ser inducidas a partir de la segunda semana posnatal, regularmente aparecen a partir de la tercera y cuarta; la salivación profusa ocurre con frecuencia. Las convulsiones tónico-clónicas disminuyen en frecuencia conforme aumenta la edad. Usualmente, las convulsiones ocurren dentro de los primero 60 minutos después de la administración de AK (Velísek 2006).

En relación a los patrones EEG se han observado espigas, espigas-ondas y poliespigas (Figura 3). Un estudio en ratas con electrodos intra-hipocámpales y subdurales, muestra espigas durante el $\mathrm{SE}$, las crisis electrográficas convulsivas y no convulsivas ocurren en los circuitos hipocámpicos y corticales, sin embargo, la actividad electrográfica convulsiva y el presunto daño neuronal es difuso, (Heillier et al. 1999). Los registros hipocámpicos pueden presentar ondas dentadas (Velísek 2006). 
Sin embargo, existe una pobre correlación entre la actividad motora y EEG inducida por AK ya que la actividad epileptiforme observada en el EEG pue-de durar horas después de que las convulsiones se hayan detenido (Giorgi et al. 2005; Velísek 2006).

De igual forma, estudios metabólicos han demostrado en este modelo un incremento en el consumo de glucosa en la formación hipocámpica, el septum lateral, sustancia negra pars reticulata, putamen, núcleo talámico anterolateral, núcleo accumbens, complejo rostral límbico, corteza perirrinal, corteza piriforme y amígdala (Albala et al. 1984; Ben-Ari et al. 1981, 1984). Por el contrario, en neocorteza, prosencéfalo, mesencéfalo y puente se presenta una disminución en el consumo de glucosa (Velísek 2006).

\section{Conclusiones}

EI SE representa una emergencia neurológica con consecuencia a largo plazo, la clasificación tiene diversos propósitos: facilitar la comunicación entre el equipo médico al establecer un lenguaje "común" y además permite mejorar el abordaje terapéutico de los pacientes, basado en el actual entendimiento de la patofisiología, pronóstico, etiología y edad; finalmente permite el desarrollo de estudios epidemiológicos del SE que puedan diri-gir mejores estrategias de prevención y dar un seguimiento a las consecuencias de largo plazo. Por otra parte, los modelos animales nos permiten mimetizar los diversos mecanismos histopatológicos que subyacen al SE, dando pauta a la investigación clínica para el desarrollo de nuevas estrategias terapéutica.

\section{Literatura citada}

Albala, B.J., Moshé, S.L. y Okada, R. Kainic-acid-induced sei-zures: a developmental study. Dev Brain Res. 1984. 13: 139-148.

Atmaca M., Bebek N., Bayakan B., Gokyigit A. y Gurses C. Predictors of outcomes and refractoriness in status epilepticus: a prospective study. Epilepsy \& Behavior 75(2017)158-164.

Barzegar M., Mahdavi M., Zalegolab A. y Tabrizi A. Refractory Convulsive Status Epilepticus in Children: Etiology, Associated Risk Factors and Outcome. Iran J Child Neurol.
Autumn 2015;9(4): 24-31.

Bauer, G, Trinka, E. Nonconvulsive status epilepticus and coma. Epilepsia 2010; 51: 177- 190.

Ben-Ari, Y., Riche, D., Temblay, E. y Charton, G. Alteration in local glucose consumption following systemic adminis-tration of kainic acid, bicuculine or metrazol. Eur Neurol. 1981a. 20_173-175.

Ben-Ari, Y., Temblay, E., Riche, D., Ghilini, G. y Naquet, R. Electrographic, clinical and pathological alterations following systemic admnistration of kainic acid, bicuculine or pentetrazole: Metabolic mapping using the deoxyglucose method with special reference to the pathology of epi-lepsy. Neuroscience. 1981b. 6: 1361-1391.

Blumenfeld, $H$. From molecules to networks: cortical/subcortical interactions in the pathophysiology of idiopathic generalized epilepsy. Epilepsia. 2003. 44:7-15.

Brabcová, R., Kubová, H., Velisek, L. y Mares, P. Effects of benzodiazepine, bretazenil (Ro 16-6028), on rhythmic metrazol EEG activity: comparison with standar convulsivant. Epilepsia. 1993. 34: 1135-1140.

Bragin, A., Mody, I., Wilson, C.L. y Engel, J.Jr. Local genera-tion of fast ripples in epileptic brain. J Neurosci. 2002. 22: 2012-2021.

Bragin, A. Engel, J.Jr., Wilson, C.L., Vizentin, E. $y$ Mathern, G.W. Electrophysiologic analysis of chronic seizures model af-ter unilateral hippocampal KA injection. Epilepsi. 1999. 40: 1210-1221.

Browing RA. Role of the brain-stem reticular formation in to-nicclonic seizures: lesion and pharmacological studies. Federation Proceedings 1985. 44, 2425-31.

Buzsaki, G., Horvath, Z. Urioste, R., Hetke, J. y Wise, K. Hi-gh-frequency network oscillation in the hippocampus. Science. 1992. 256: 1025-1027.

Cherubini, E., DeFeo, M.R., Mecareli,, O. y Ricci, G.F. Behavio-ral and electrographic patterns induced by systemic ad-ministration of kainic acid in developing rats. Dev Brain Res. 1983. 9: 69-77.

Cortez, M.A., Snead III, O.C. Pharmacologic models of genera-lized absence seizures in rodents. Pitkänen, A., Schwart-zkroin, P.A. y Moshé, S.L. (Ed.). Models of Seizures and Epilepsy. Elsevier. 2006. 111-126.

Cuero M. y Varelas P. Super-Refractory Status Epilepticus. Curr Neurol Neurosci Rep (2015) 15: 74.

Curia, G., Longo, D., Biagini, G., Jones, R.S., Avoli. M. The pilo-carpine model of temporal lobe epilepsy. J Neurosci Me-thods. 2008. 172:143-57.

Datar S. New developments in refractory status epilepticus. Neurol Clin 35(2017)751-760.

De Casrilevitz, M., Engelhardt, E. Y Esbérard, C. Maduration of convulsogenic activity induced by leptazol in the albino rat. Br J Pharmacol. 1971. 42: 31-42.

Delaj L., Novy J., Ryvlin P., Marchi N. y Rossetti A. 2016. Re-fractory and super-refractory status epilepticus in adults: a 9-year cohort study. Acta Neurol Scand.

Dichter, M.A. Emerging concepts in the pathogenesis of epilepsy and epileptogenesis. Arch. Neurol. 2009. 66:443- 
447.

Dudek, F.E., Clark, S., Williams, P.A. y Grabenstatter, H. Kai-nate-induced status epilepticus: a chronic model of acquiered epilepsy. Pitkänen, A., Schwartzkroin, P.A. y Moshé, S.L. (Ed.). Models of Seizures and Epilepsy. Else-vier. 2006. 415-432.

Engel, J.Jr. Intractable epilepsy: definition and neurobiology. Epilepsia. 2001. 42.

Engel, J.Jr., Schwartzkroin, P.A. 2006. What should be mo-del? pp- 1-14. En: Pitkanen A., Schwartkroin, P.A., Mos-he, S.L., Eds.; Models of Seizures and Epilepsy. Elsevier Academic Press, New York, USA. 712 pp. [978-0-12-088554-1]

Erdogan, H., Ekici, F., Katar, M., Kesici, H. y Aslan, H. The pro-tective effect of endothelin- $A$ receptor antagonist $B Q-123$ in pentylenetetrazole-induced seizures in ratas. Hu-man \& Experimental Toxicol. 2014. 33: 1-9.

Fernandes, M.J.S., Naffah-Mazzacoratti, M.G. y Cavelheiro, E.A. 1996. Na+K+ ATPasa in the rat hippocampus: $A$ study in pilocarpine model of epilepsy. Neurochem Int. 28: 497-500

Fisher, R.S., Van Emde Boas, W., Blume, W., Elger, C., Gen-ton, P., Lee, P. y Engel, J.Jr. Epileptic seizures and epilepsy: definitions proposed by th International Ligue Against Epilpesy (ILAE) and the international Bureau of Epilepsy (IBE). Epilepsia. 2005. 46: 470-472.

Fisher, R.S. Animal models of epilepsies. Brain Res. 1989. 14: 245-278.

Giorgi, F.S., Malhotra, S., Hasson, H., Velísková, J., Rosenbaum, D. y Moshé, S.L., Effects of status epilepticus early in life on susceptibility to ischemic injury in adulthood. Epilep-sia. 2005. 46: 490-498.

Grenier, F., Timofeev, I. Y Steriade, M. Focal synchronization of ripples $(80-200 \mathrm{~Hz})$ in neocortex and their neuronal correlates. J Neurophysiol. 2001. 86: 1884-1898.

Hauser, W.A. Status epilepticus: epidemiologic considerations. Neurol. 1990 40: 9-13.

Hansen, S.L., Sperling, B.B. y Sánchez, C. Anticonvulsivants and antiepileptogenic effects of GABAA receptor li-gands in pentylen-etetrazolekindled-mice. Progress in Neuropsychipharmacol Biological Psychiatry. 2004. 28: 105-113.

Heillier, J.L., Patrylo, P.R., Dou, P., Nett, M., Rose, G.M. $y$ Dudek, F.E. Assesment of inhibition and epileptiform activity in the septal dental gyrus of freely behaving rats during the first week after kainate treatment. J Neurosci. 1999. 31: 73-84.

Hernández-Domínguez J., Cano-Salazar A., CalderónVallejo A., Cuevas-García C. Experiencia del estado epiléptico en 5 años en el servicio de Neurología del Hospital de Especialidades del CMN SXXI. Rev Med Inst Mex Seguro Soc. 2017;55 Supl 4:S369-74.

Hesdorffer, D.C., Logroscino, G., Cascino, G., Annegers, J.F. y Hauser, W.A. Risk of unprovoked seizure after acute symptomatic seizure: effect of status epilepticus. Ann
Neurol. 1998. 44: 908-912.

Kupferberg, $\mathrm{H}$. Animal models used in the screening of an-tiepileptic drugs. Epilepsia. 2001. 42: 7-12.

Mares, P., Maresová, D. y Schickerová, R. Effect of antiepilep-tic drugs on metrazol convulsions during ontogenesis in rats. Physiol Bohemoslov. 1981. 30

McCandless, D.W. Epilepsy, Animal and Human Correlations. Springer (Ed). 2012.

Trinka E, Höfler J, Zerbs A. Causes of status epilepticus. Epi-lepsia. 2012. 53:127-38.

Medina-Ceja, L., Morales-Villagran, A. y Tapia, R. Action of 4-aminopyridine on extracelular amino acids in hippo-campus and enthorhinal cortex: a dual microdialysis and electroencephalograpic study in awake rats. Brain Res Bull. 2000. 53: 255-262.

Miller, JW. Functional atomy of pentilenetetrazol and electros-hock seizures in the rat brainstem. Ann Neurol 1987. 22, 615-21.

Miller, L.P., Jhonson, A.E., Gelhard, R.E. e Insel, T.R. The on-togeny of excitatory amino acid receptors in the rat fo-rebrain-II. Kainic acid receptors. Neuroscience. 1990. 35: 45-51.

Nadler, J.V. Minireview: Kainic acid as tool for the study of temporal lobe epilepsy. Life Sci. 1981. 29: 2031-2042.

Olsen, R.W. The GABA postsynaptic membrane receptor-ionophore complex. Site of action of convusivant and anticonvulsant drugs. Mol Cell Biochem. 1981. 39: 261-279.

Owolabi L., Ibrahim A., Datti A. y Owolabi S. Status epilepti-cus in adults: a study from Nigeria. International Journal of Epilepsy I (2014)69-74.

Pirttila, T.R., Pitkanen, A., Tuunanen, J. y Kauppinen, R.A. Ex vivo MR microimaging of neuronal damage after kai-nate-induced status epilepticus in rat: correlation with quantitative histology. Mgn Reson Med. 2001. 46: 946-954.

Ramanjaneyulu, R., Ticku, M.K. Interactions of pentamethyle-netetrazole and tetrazole analogues with picrotoxin site of benzodiazepine-GABA receptorinophore complex. Eur J Pharmacol. 1984. 98: 337-345.

Reinhard, J.F. y Reinhard, J.F.J. Experimental evaluation of an-ticonvulsivants. Vida, J.A. (Ed). Anticonvulsivants. New York: Academic Press. 1977. 57-111.

Riban, V., Bouilleret, V., Pham, L., Fritschy, J.M., Marescaux, C. Y Depaulis, A. Evolution of hippocampal epileptic activi-ty during the development of hippocampal sclerosis in a mouse model of temporal lobe epilepsy. Neurosc. 2002. 112: 101-111.

Rowley, H.L., Marsden, C.A. y Martin, K.F. Differential effects of phenytoin and sodium valproate on seizure-induced changes in gamma-aminobutyric acid and glutamate release in vivo. Eur J Pharmacol. 1995. 294: 541-546.

Sampieri, A., Rivera-Espinoza, L., Zavala-Tecuapetla, C. Y Carmona-Aparicio, L. Modelos experimentales de la epi-lepsia del lóbulo temporal. Pediatr Mex. 2011. 32: 311-312. 
Stafstrom, C. E. Pathophisiologycal mechanisms of seizures and epilepsy: A primer. In Epilepsy mechanism, models and translational perspectives. J.M.Rho, R. Sankar, Stafstrom, C.E. eds. 2010. pp-3-20.

Swinyard, E.A., Woodhead, J.H., White H.S. y Franklin, M.R. Experimental selection, quantification, and evaluation of antivonculsants. Levy, R.H., Dreifuss, F.E., Matsson, R.H., Meldrum, B.S. y Penry, J.K. (Ed). Antiepileptic drugs. New York: Raven Press. 1989. 85-102.

Tapia, R., Medina-Ceja, L. y Pena, F. On the relationship between extracelular glutamate, hyperexcitation and neurodegeneration, in vivo. Neurochem Int. 1999. 34: 23-31.

Tremblay, E., Nitecka, L., Berger, M.L. y Ben Ari, Y. Maturation of kainic acid seizure-brain damage syndrome in the rat.l. Clinical. Electrographic and metabolic observations. Neurosci. 1984. 13: 1051-1072.

Trinka, E.; Cock, H.; Hesdorffer, D.; Rossetti, A. O.; Scheffer, I. E.; Shinnar, S.; Shorvon, S.; Lowenstein, D. H. A definition and classification of status epilepticus - Report of the ILAE Task Force on Classification of Status Epilepticus. Epilepsia. 2015. 56: 1515-1523.

Velísek, L. Models of chemically-induced acute seizures. Pitkänen, A., Schwartzkroin, P.A. y Moshé, S.L. (Ed.). Models of Seizures and Epilepsy. Elsevier. 2006. 127152.

Velísek, L., Kubová, H., Pohl, M., Stanková, L., Mares, P., Schickerová, R. Pentylenetetrazol-induced seizures in rats: An ontogenetic study. Naunyn Schmiedebergs Arch Pharmacol. 1992. 346: 588-591.

Velísková, J., Velísek, L. y Mares, P. Epileptic phenomena produced by kainic acid in laboratory rats during ontogenesis. Physiol Bohemoslov. 1988. 37: 395-405.

Vernadakis, A., Woodbury, D.M. The developing animal as a model. Jasper, H., Ward, A., Pope, A. (Ed). Mechanisms of epilepsies. Boston: Little, Brown. 1969a. 535-541.

Vernadakis, A., Woodbury, D.M. Maturational factors in development of seizures. Epilepsia. 1969b.

Villalpando-Vargas, F., Medina-Ceja, L. Effect of sparteine on Status Epilepticus induced in rats by Pentylenetetrazole, Pilocarpine and Kainic Acid. Brain Research. 2015. 1624: 59-76.

Weller, A., Mostofsky, D.I. Ontogenic development and pentylenetetrazol seizure thresholds in rats. Physiol Behav. 1995. 57: 629-631.

Ylinen, A., Bragin, A., Nadasdy, Z., Jando, G., Szabo, I., Sik, A. y Buzsaki, G. Sharp wave-associated high-frecuency oscillation $(200 \mathrm{~Hz})$ in the intact hippocampus: network and intracelular mechanism. J Neurosci. 1995. 15: 3046.

Yonekawa WD. Relation between pentilenetetrazole-induced seizures and brain pentilenetetrazol levels in mice. J Pharmacol Exp Ther 1980. 214,589-3.

Zhao, Q., Holmes, G.L. Repetitive seizures in the immature brain. Pitkänen, A., Schwartzkroin, P.A. y Moshé, S.L. (Ed.). Models of Seizures and Epilepsy. Elsevier. 2006. 341-350. 